\title{
IMPACTO DE LA ESPERANZA EN LAS ACTITUDES POLÍTICAS EN HABITANTES DE LA CIUDAD DE AREQUIPA
}

WALTER ARIAS

https://orcid.org/0000-0002-4183-5093

JULIO CÉSAR HuAMANI

https://orcid.org/0000-0001-8159-803X

Karla Ceballos

https://orcid.org/0000-0001-8006-3738
CAMILA CÁRDENAS

Ximena Tamayo Butilier

Eduardo Marquina Carrasco

MaRcia díAZ CANO

Universidad Católica San Pablo, Arequipa, Perú

Recibido: 8 de julio del 2019 / Aceptado: 14 de agosto del 2019

doi: 10.26439/persona2019.n022(2).4563

Resumen. Se analiza el impacto de la esperanza en las actitudes hacia la política entre los habitantes de la ciudad de Arequipa, Perú, aplicando el Cuestionario de Actitudes hacia la Política y el Índice de Esperanza de Herth (HHI). La muestra probabilística por racimos estuvo compuesta por 875 personas ( $47,54 \%$ varones y $52,46 \%$ mujeres), con una edad promedio de 38 años, residentes en once distritos de la ciudad de Arequipa. Los datos fueron procesados en forma descriptiva, comparativa y correlacional. Los resultados indican que, en función del grado de instrucción, tanto la esperanza como las actitudes hacia la política presentan diferencias significativas.

Palabras clave: esperanza / actitudes / participación política / psicología positiva

\section{THE IMPACT OF HOPE ON ATTITUDES TOWARD POLITICS AMONG RESIDENTS OF THE CITY OF AREQUIPA}

\begin{abstract}
This research analyzes the impact of hope on attitudes toward politics among residents of the city of Arequipa, Peru. The Attitudes Toward Politics Questionnaire and the Herth Hope Index ( $\mathrm{HHI}$ ) were administered to a cluster sample consisting of 875 adults (47,54 \% males and 52,46\% females) with an average age of 38 years who lived in eleven districts of the city of Arequipa. Data was processed using a descriptive, comparative and correlational design. The results show that hope and attitudes toward politics differ significantly according to the education level.
\end{abstract}

Keywords: hope / attitudes / political participation / positive psychology 


\section{INTRODUCCIÓN}

Los primeros trabajos de psicología política se remontan a la primera década del siglo xx con Gustav Le Bon, pero recién en la década de 1970 se puede distinguir como disciplina autónoma (Dávila, Fouce, Gutiérrez, Lillo y Martín, 1998). Así, surgida de la psicología social (Ibáñez, 1993), la psicología política se ha focalizado en temas como la interacción de los procesos psicológicos y políticos, el carácter histórico de la conducta sociopolítica, la personalidad y el liderazgo político, la influencia social y la comunicación persuasiva, las ideologías políticas, etcétera (Montero y Dorna, 1993).

De este modo, la psicología política puede definirse como un campo dentro de la psicología que está abocado al estudio de "los patrones de pensamiento político, sentimientos e identidad, así como la interacción de estos patrones y su impacto en la elección política, y otras formas de comportamiento político" (Temkin y Flores, 2011, p. 69). Asimismo, la psicología política ha recibido la influencia inicial del psicoanálisis (Jiménez, 1993), para luego convertirse en una disciplina impregnada de postulados cognitivo-sociales (Dávila et al., 1998). La psicología positiva, por su parte, no ha desarrollado líneas sistemáticas de trabajo en esta rama (Arias, 2016), pues se han privilegiado más la psicología clínica, la psicología educativa y la psicología organizacional (Park, Peterson y Sun, 2013).

En ese sentido, la presente investigación busca valorar el impacto de la esperanza y otras variables demográficas en las actitudes hacia la política de una muestra de habitantes de la ciudad de Arequipa, ubicada al sur del Perú. No existen estudios nacionales ni internacionales que aborden ambas variables desde el enfoque de la psicología positiva, por lo que este trabajo reviste relevancia teórica y metodológica, así como originalidad, al ubicarse dentro del campo de la psicología política positiva.

La esperanza, por un lado, podría definirse como una virtud trascendente (Peterson y Seligman, 2004), lo que ha sido tema de estudio de autores como Erich Fromm (2012) o Viktor Frankl (2002), pero solo con la psicología positiva ha sido objeto de teorizaciones que han permitido valorar sus aplicaciones en el campo de la salud y la psicología clínica (Snyder, Rand y Sigmon, 2002; Cheavens, Feldman, Woodward y Snyder, 2006; Cleary, Sayers y López, 2016), la psicología educativa (López, Sage, Robinson, Marques y Ribeiro, 2014) y la psicología organizacional (Malik, 2013).

Teóricamente, Snyder (2000) considera que la esperanza comprende metas, agencias y vías. Las metas son objetivos mentales que implican secuencias de acciones; las vías son las rutas de acción que las personas perciben como viables para conseguir lo que desean; mientras que las agencias hacen referencia a las capacidades que se posee para conseguir las metas deseadas (Snyder, Rand y Sigmon, 2002). Por otro lado, para Dufault y Martocchio (1985), la esperanza es una fuerza vital dinámica y multidimensional que se caracteriza por la confianza de alcanzar en el futuro las 
metas planteadas. Dichos autores plantearon seis dimensiones de la esperanza, distribuidas en los ámbitos afectivo, cognitivo, conductual, afiliativo, temporal y contextual. Sobre la base de estas ideas, Herth (1991) desarrolló un modelo de tres dimensiones: cognitivo-temporal, como valoraciones realistas de los resultados deseados; afectivoconductual, como sentimientos de confianza necesarios para emprender acciones, y afiliativo-contextual, como el vínculo que se establece entre una persona y las demás (Martínez, Cassaretto y Herth, 2012).

En Latinoamérica, son pocos los estudios sobre la esperanza, pero se puede destacar la investigación de Romagosa y Albizú-Miranda (1986), quienes tempranamente, a mediados de la década de 1980, encontraron que la fe, el amor y la esperanza se relacionan con la salud mental. En México, se reportó que los varones poseen mayores niveles de esperanza que las mujeres (Valdez et al., 2014). Asimismo, en Brasil se han realizado adaptaciones psicométricas del Índice de Esperanza de Herth (Sartore, Alvez y Herth, 2010), al igual que en el Perú (Martínez, Cassaretto y Herth, 2012). También se puede destacar el estudio de Yager-Elorriaga, Berenson y McWhirter (2014), quienes encontraron en una muestra de latinos residentes en Estados Unidos que el orgullo étnico correlacionaba positiva y significativamente con la esperanza.

En el Perú, donde se ubica el presente trabajo, los primeros estudios sobre la esperanza corresponden a investigaciones de corte psicométrico realizadas por Arnau et al. (2010) y Martínez, Cassaretto y Herth (2012), quienes adaptaron la versión completa de la Escala de Esperanza de Herth en muestras de estudiantes universitarios. Asimismo, se han usado medidas de esperanza y bienestar psicológico para obtener evidencias de validez convergente de la Escala de Sentido del Humor de Martin et al. (Cassaretto y Martínez, 2009), y para analizar las propiedades psicométricas del inventario Razones para Vivir, en estudiantes universitarios (Cassaretto y Martínez, 2012).

Con respecto al Herth Hope Index, Castilla, Urrutia, Shimabukuro y Caycho (2014), luego de analizar sus propiedades psicométricas en 210 estudiantes universitarios de la ciudad de Lima, reportaron la existencia de dos factores, identificados como optimismo/ soporte y agencia, con una elevada consistencia interna $(\alpha=0,851)$. Con este mismo instrumento, se ha reportado que, en una muestra de 420 personas entre los 16 y los 38 años de edad, las mujeres obtienen puntuaciones más altas en esperanza, así como quienes tienen más edad (Caycho, Castilla y Ventura-León, 2016).

Para el caso de la psicología política, los primeros estudios realizados en el Perú corresponden a Carlos Franco en la década de 1980 (Franco, 1980), y en la siguiente década surgieron estudios sobre identidad nacional (Salgado, 1996, 1997), orientación a la dominancia social (León y León, 2014), discriminación y conflictos sociales (Rottenbacher, 2015; Rottenbacher y De la Cruz, 2012), aspectos psicológicos durante los procesos electorales (Espinosa, 2008; Guevara y Espinosa, 2014), nacionalismo y 
autoconcepto (Espinosa et al., 2016), bienestar subjetivo, social y colectivo (Espinosa y Tapia, 2011; Espinosa, Beramendi y Zubieta, 2015; Espinosa, Freire y Ferrándiz, 2016).

Ahora bien, la psicología política en el Perú se ha desarrollado en los últimos años (Espinosa, Cueto y Schmitz, 2012), pero no lo suficiente como para ubicarse en el nivel de España, Colombia, México, Argentina o Brasil, países que mantienen el liderazgo de la investigación en psicología política en Iberoamérica (Polo, Godoy, Imhoff y Brussino, 2014). Sin embargo, ha seguido el mismo proceso de desarrollo, ya que surge como campo independiente en la década de 1980, al igual que en otros países de la región (Rodríguez, 2001). Además, pueden distinguirse las mismas etapas, características y modos de hacer psicología política (Montero, 1991).

Por otro lado, la psicología política latinoamericana aún enfrenta una diversidad de problemáticas epistemológicas y socioculturales determinantes en su desarrollo: el papel de la ideología en la construcción del conocimiento (Buss, 1979), la dimensión individualismo-colectivismo (Morales, Gaviria, Molero, Arias y Páez, 2000), la dicotomía entre lo individual y lo público (Quintana y Curbelo, 2015), la comunión entre lo ético y lo político (Silva, 2013) y la inserción de la psicología positiva en la psicología política (Mentinis, 2011), entre otras cuestiones.

Con respecto a las actitudes políticas, pueden definirse como las orientaciones adquiridas, que son relativamente estables e inciden directamente en el comportamiento político (Chacón y Alvarado, 2007). De acuerdo con un estudio previo, se ha podido determinar que las actitudes hacia la política tienen dos dimensiones: la participación política y la gobernabilidad (Arias et al., 2017). Es decir que las actitudes hacia la política están determinadas por la eficacia en la capacidad de gobierno y la participación de sus ciudadanos.

En cuanto a la participación política, se trata de un fenómeno eminentemente social (Sorribas y Brussino, 2013) que se entiende como "aquellas intenciones legales o no, desarrolladas por individuos y grupos con el objetivo de apoyar o cuestionar a cualquiera de los distintos elementos que configuran el ámbito de lo político" (Sabucedo, 1996, citado por Sabucedo y Fernández, 2001, p. 70). Sin embargo, la participación política usualmente se reduce a la conducta de voto (Rodríguez, 2004), y está influida por diversos factores, como variables demográficas, los valores, las ideologías y la personalidad (Rodríguez et al., 1996).

Por ejemplo, se ha reportado que las mujeres son menos liberales que los varones, y que los de mayor edad son más conservadores (González y Darias, 1998). Asimismo, a mayor formación política y nivel socioeconómico, mayor es la participación política (Sorribas y Brussino, 2013). En ese sentido, las personas de nivel socioeconómico bajo forjan una percepción social negativa (Sabucedo y Fernández, 2001), lo que se explica 
porque se sienten impotentes frente al escenario político y sus implicancias, dada su escasa capacidad de decisión y sus sentimientos de indefensión (Rodríguez et al., 1996). Esto podría tener cierta relación con la esperanza, ya que, mientras menor sea el nivel socioeconómico de las personas, menor sería su esperanza y su participación política.

La afiliación política (Gattino, Roccato y Tamagnone, 2002) y los valores (Schwartz y Barnea, 1995) también tienen cierta injerencia en la participación política y la gobernabilidad. En ese sentido, la participación política se relaciona con la gobernabilidad, porque se refiere a los comportamientos que afectan las decisiones del gobierno (Delfino y Zubieta, 2014). Cuando se percibe favorablemente el gobierno, hay mayores niveles de satisfacción y la participación política aumenta (Peterson, 1991).

Valores y virtudes como la esperanza, la participación política y la gobernabilidad se relacionan con el civismo (Pasquino, 2001). Parece ser que la esperanza otorga sentido y satisfacción con la vida, lo que bien puede ser una condición esencial para el desarrollo de actitudes favorables hacia la política (Bronk, Hill, Lapsley, Talib y Finch, 2009). De este modo, las actitudes que definen la participación política son la implicación política, el deber cívico, la politización y la satisfacción política (Chacón y Alvarado, 2007), mientras que, por otro lado, la participación política se relaciona con el bienestar psicosocial (Eiroa, 2013).

En nuestro país, empero, existe una escasa participación política mediada por la insatisfacción de la población con el quehacer de la clase política, así como por las condiciones de corrupción, inseguridad y desigualdad socioeconómica. Por ello, pensamos que entre esperanza y actitudes hacia la política no existirán relaciones positivas, y que variables demográficas como la edad y el grado de instrucción tendrán efecto en la esperanza y la participación política de los habitantes de la ciudad de Arequipa.

\section{MÉTODO}

\section{Participantes}

La muestra está constituida por 875 personas con una edad media de 38,26 años y una desviación estándar de $\pm 16,35$. El 47,54 \% son varones y el $52,46 \%$ son mujeres. El 39,08 \% son solteros, el 42,17\% son casados, el 8,8 \% son convivientes, el 6,05\% son divorciados y el $3,88 \%$ son viudos. El 4,45 \% tiene estudios primarios, el 19,31\% estudios secundarios, el 15,77 \% tiene formación técnica de nivel superior, el 56,45\% tiene estudios universitarios y el 3,88 \% tiene estudios de posgrado. Los participantes provienen de once distritos de la ciudad de Arequipa, al sur del Perú. El método de selección de la muestra fue probabilístico, mediante la técnica de muestreo por racimos (Hernández, Fernández y Baptista, 2006). 


\section{Instrumentos}

El Cuestionario de actitudes hacia la política fue diseñado por Arias et al. (2017) con la finalidad de valorar las actitudes hacia la política en la población de la ciudad de Arequipa. Consta de once ítems que tienen cinco alternativas de respuesta en una escala tipo Likert que comprende los siguientes niveles: Nada (1), Poco (2), Algo (3), Mucho (4) y Todo (5). Tiene dos factores: gobernabilidad y participación política, que explican el $41,7 \%$ de la varianza total y cuentan índices de validez y confiabilidad adecuados $(\alpha=0,745$ y $\alpha=0.730$, respectivamente), obtenidos mediante análisis factorial exploratorio y el método de consistencia interna con la prueba Alfa de Cronbach.

Se utilizó, además, el Índice de esperanza de Herth (HHI, 1992), derivado de la versión original de treinta ítems (Herth, 1991). El HHI consta de doce ítems con cinco alternativas de respuesta en una escala tipo Likert (Totalmente en desacuerdo a Totalmente de acuerdo), y está validado por Castilla et al. (2014) para población universitaria de la ciudad de Lima (Perú). Dicha versión cuenta con un índice de confiabilidad de 0,851 obtenido por medio del método de consistencia interna con la prueba alfa de Cronbach, correlaciones ítem-test positivas y altamente significativas, así como una estructura factorial de dos dimensiones, obtenidas mediante el método de componentes principales y rotación Varimax. Sin embargo, en un estudio previo, hemos determinado las propiedades psicométricas para dicho instrumento en la población arequipeña, con una estructura unidimensional y altos índices de confiabilidad obtenidos con el coeficiente Omega, que superan el 0,8 (Arias et al., 2018).

\section{Procedimiento}

El recojo de los datos se hizo en horas del día, siguiendo los lineamientos del muestreo explicados anteriormente. Las personas evaluadas firmaron un consentimiento informado y recibieron orientación adecuada para el llenado de los instrumentos. Los datos fueron procesados con el programa SPSS versión 21 y el Amos 22, según el nivel de medición de las variables y los fines del estudio.

\section{RESULTADOS}

En la tabla 1 se muestran los resultados descriptivos, donde se observa que en la variable esperanza $(M=37,22 ; D E= \pm 4,10)$, con un puntaje mínimo de 13 y máximo de 48 , indicaría puntuaciones altas. En cuanto a las actitudes hacia la política, la dimensión gobernabilidad $(M=16,23 ; D E= \pm 4,53)$, con un puntaje mínimo de 7 y máximo de 48 , indicaría puntuaciones promedio, mientras que la dimensión participación política $(M=12,69 ; D E= \pm 3,06)$, con un puntaje mínimo de 4 y máximo de 20 , indicaría puntuaciones con tendencia alta. Asimismo, la puntuación total de actitud hacia la política (M $=28,92 ; \mathrm{DE}= \pm 6,29$ ) tiene puntajes altos. 
Tabla 1

Análisis descriptivo de las variables

\begin{tabular}{lcccc}
\hline Estadísticos & Esperanza & Gobernabilidad & $\begin{array}{c}\text { Participación } \\
\text { política }\end{array}$ & $\begin{array}{c}\text { Actitud hacia } \\
\text { la política }\end{array}$ \\
\hline Media & 37,22 & 16,23 & 12,69 & 28,92 \\
Mediana & 37 & 16 & 13 & 29 \\
Moda & 34 & 16 & 13 & 30 \\
Desviación estándar & 4,10 & 4,53 & 3,06 & 6,29 \\
Varianza & 16,84 & 20,55 & 9,37 & 39,56 \\
Asimetría & -.925 & .129 & -.141 & -.048 \\
Curtosis & 3,551 & -.452 & -.077 & -.033 \\
Mínimo & 13 & 7 & 4 & 11 \\
Máximo & 48 & 32 & 20 & 47 \\
IC (95 \%) & 36,94 & 15,93 & 12,49 & 28,5 \\
& 37,49 & 16,53 & 12,89 & 29,33 \\
\hline
\end{tabular}

En la tabla 2 se muestran los resultados comparativos según el grado de instrucción, el estado civil y el sexo de las personas evaluadas. Se pueden observar diferencias estadísticamente significativas en la variable esperanza con respecto al grado de instrucción $\left(F_{(875)}=8,142 ; p=.000\right)$, donde los participantes con el grado académico de maestría ( $M=40,23$ ) puntúan más alto que los participantes con grado de instrucción primaria, secundaria, técnica y universitaria $(M \geq 36,49<37,34)$. Del mismo modo, con la variable gobernabilidad $(F(875)=7,173 ; p=.000)$, los participantes con grado de instrucción técnica y universitaria ( $M=16,13$ y 17,96, respectivamente) puntúan más alto que aquellos con grado de instrucción de Maestría, Primaria y Secundaria $(M \geq 15,06<15,58)$. En la variable participación $(F(875)=6,342 ; p=.000)$, los grupos con grado de instrucción técnica, universitaria y maestría $(M \geq 12,79<13,54)$ puntúan más alto que los grupos con grado de instrucción primaria y secundaria $(M=11,15$ y 12,08, respectivamente). Asimismo, existen diferencias con la variable actitud hacia la política $(F(875)=8,045 ; p=.000)$, de modo que el grupo con grado de instrucción técnica $(M=31,25)$ puntúa más alto que los demás grupos.

En resumen, los grupos con grado de instrucción técnica, universitaria y maestría presentan puntajes más altos en las variables esperanza y actitudes hacia la política. Con respecto al estado civil, no existen diferencias significativas con ninguna variable de las actitudes hacia la política, mas sí con la variable esperanza $(F(875)=2,897$; $p=.021)$, donde los grupos con estado civil de casado, conviviente y viudo $(M \geq 37,01<37,74)$ puntúan más alto que los de estado civil divorciado y soltero $(M=36,08$ y 36,51 , respectivamente), datos que indicarían que la esperanza se presenta más en personas casadas y en 
quienes cohabitan que en personas que viven solas. Con respecto al sexo, no se encontraron diferencias significativas en este estudio.

Tabla 2

Diferencias a través del ANOVA de un factor y la T de student para muestras independientes

\begin{tabular}{lcccccc}
\hline & \multicolumn{2}{c}{ Grado de instrucción } & \multicolumn{2}{c}{ Estado civil } & \multicolumn{2}{c}{ Sexo } \\
\cline { 2 - 6 } & $\mathrm{F}$ & $\mathrm{P}$ & $\mathrm{F}$ & $\mathrm{p}$ & $\mathrm{t}$ & $\mathrm{p}$ \\
\hline Esperanza & 8,142 & .000 & 2,897 & .021 & $-1,818$ & .066 \\
Gobernabilidad & 7,173 & .000 & .223 & .926 & .469 & .639 \\
Participación & 6,342 & .000 & 595 & .666 & -.578 & .563 \\
Actitud hacia la política & 8,045 & .000 & .059 & .994 & .057 & .955 \\
\hline
\end{tabular}

En la tabla 3 se observan las correlaciones de las variables cuantitativas de este estudio, tales como la edad, la esperanza, las actitudes hacia la política y sus dimensiones. Puede verse que existe una correlación negativa débil de la edad con la gobernabilidad y la actitud hacia la política, de modo que los participantes con mayor edad presentan actitud baja hacia la gobernabilidad y hacia la política. Asimismo, existe correlación positiva débil de la esperanza con la participación política; es decir, los sujetos que asumen una actitud de participación política presentan mayores niveles de esperanza. La variable actitudes hacia la política correlaciona de manera fuerte, positiva y significativa con la gobernabilidad y la participación política, mientras que las dimensiones participación política y gobernabilidad correlacionan de manera positiva y moderada entre sí.

Tabla 3

Correlaciones entre las variables y la edad

\begin{tabular}{lccccc}
\hline Correlaciones & Edad & Esperanza & Gobernabilidad & $\begin{array}{c}\text { Participación } \\
\text { política }\end{array}$ & $\begin{array}{c}\text { Actitud hacia } \\
\text { la política }\end{array}$ \\
\hline Edad & 1 & & & & \\
Esperanza & 0,018 & 1 & 1 & & \\
Gobernabilidad & $-0,194^{* *}$ & 0,002 & $0,347^{* *}$ & 1 & \\
Participación política & -0.039 & $0,119^{* *}$ & $0,890^{* *}$ & $0,737^{* *}$ & 1 \\
Actitud hacia la política & $-0,159^{* *}$ & 0,059 & & \\
\hline
\end{tabular}

${ }^{* *} \mathrm{p}<.01$ 
Por otro lado, se propuso un modelo teórico que explicaba las variables de estudio y comprendía la edad, el sexo, el grado de instrucción y el estado civil. El modelo teórico propuesto se aprecia en la figura 1.

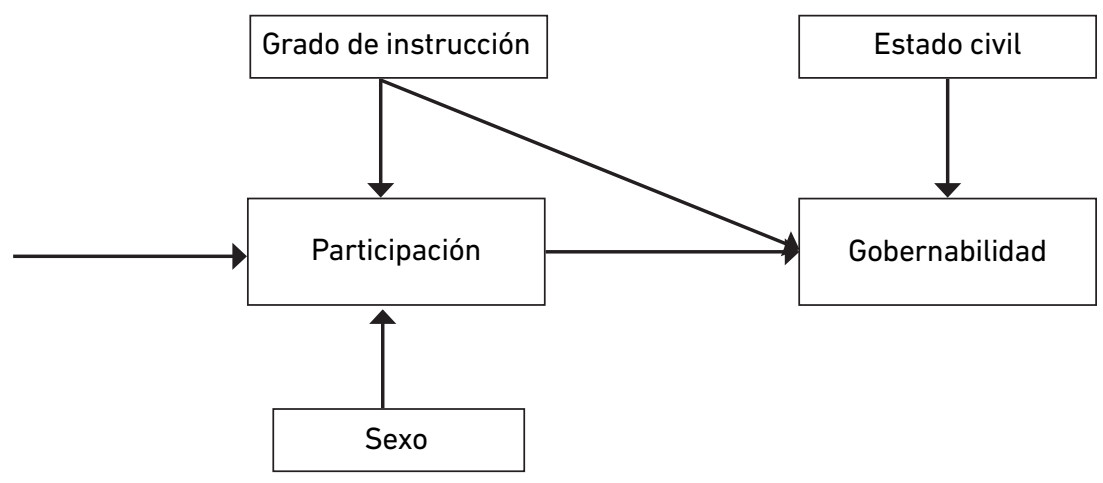

Figura 1. Modelo teórico inicial del impacto de la esperanza sobre las actitudes hacia la política teniendo en cuenta los datos demográficos.

Así, con el objetivo de contrastar el modelo teórico explicativo propuesto, se realizó un análisis path, para lo cual se valoró si posee la suficiente información en los datos muestrales para estimar los parámetros del modelo especificado. Se encontró que el modelo se encuentra sobreidentificado ( $\mathrm{gl}>6$ ), lo que implica que existe más información que parámetros a estimar y, en consecuencia, el modelo puede ser estimado y contrastado (Arbuckle, 2003).

Los resultados del modelo indican que el efecto directo más relevante sobre la gobernabilidad fue la participación, con un coeficiente Path significativo (.52; $p<0.000)$. Asimismo, el grado de instrucción tiene efecto directo sobre la participación (.35; $p<0.001)$ y la esperanza tiene un efecto directo bajo sobre la participación política $(.08 ; p<.001)$. El conjunto del modelo obtuvo un $\mathrm{R}^{2}=0,027$ para participación y $\mathrm{R}^{2}=0,123$ para gobernabilidad, es decir, el impacto de la esperanza y la participación política explican el $12 \%$ de la variabilidad de la variable gobernabilidad, con índices de ajuste adecuados. El efecto total de la esperanza fue bajo $(0,08+0,52 \times 0,35=0,21)$, sin embargo, supera el efecto directo de la esperanza sobre la participación política.

Examinando los paths (caminos) con coeficientes beta elevados que expliquen las actitudes hacia la política, la secuencia sería la siguiente: las variables intercorrelacionadas esperanza y grado de instrucción $(0,52, p=.000)$, a través del grado de participación política tienen poder explicativo sobre la gobernabilidad. 


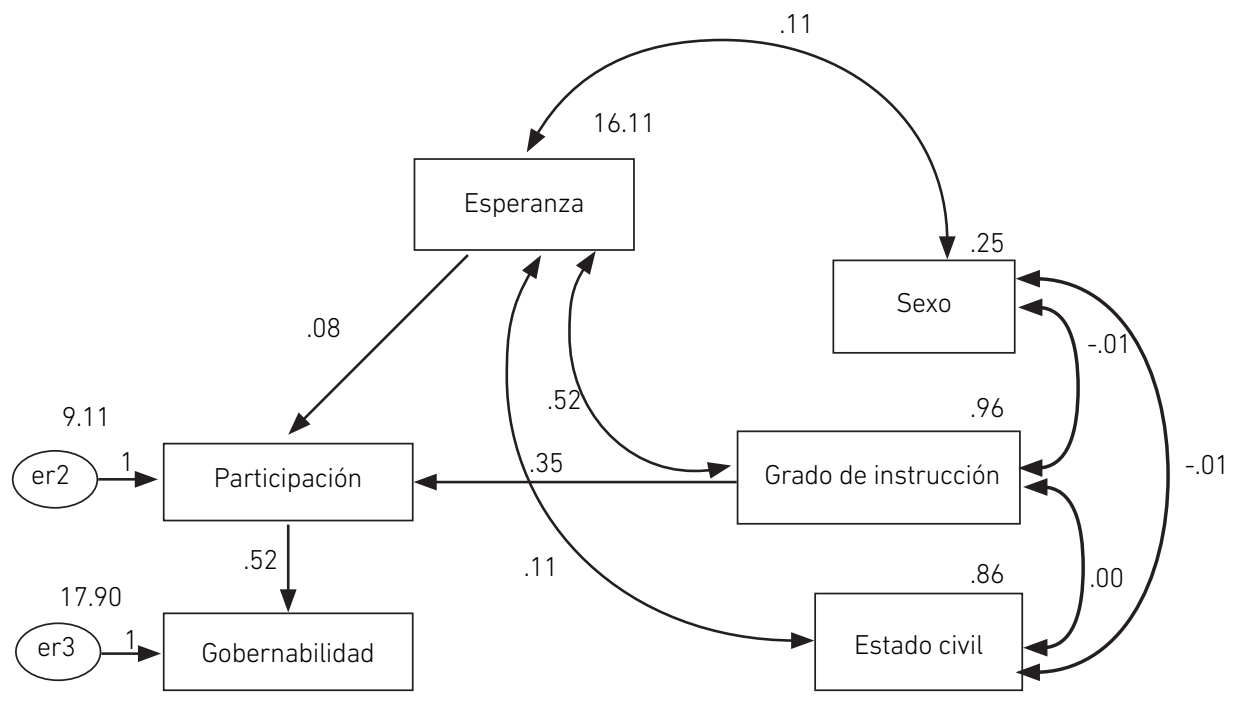

Figura 2. Path analysis estimado del impacto de la esperanza en las actitudes hacia la política en habitantes de la ciudad de Arequipa

Nota: $X^{2}=5,354 ; X^{2} / \mathrm{gl}=.892 ; \mathrm{NFI}=.961 ; \mathrm{GFI}=.998: \mathrm{CFI}=1,000 ; \mathrm{RMSEA}=.000$

\section{DISCUSIÓN}

El escenario político en el Perú, como en el de muchos países de América Latina, es de una permanente insatisfacción política debido a los actos de corrupción en los que se ven envueltos los actores políticos y los servidores públicos (Goertzel, 2005; Riesco, Alpaca y Arias, 2015), las desigualdades económicas entre los diferentes sectores sociales de la población (Ganoza y Stiglich, 2015), la inseguridad que se vivencia de manera cada vez más angustiante (Dargent, 2015) y la falta de una clase política con convicciones claras (Arellano, 2015). Todo esto ha degenerado en "bandidocracias", transfuguismo y oportunismo político, que han mellado la confianza del votante en los candidatos que se postulan a cargos políticos (Gordillo, Arana, Mestas y Salvador, 2012) y obligan a la población a elegir por el mal menor (Espinosa, 2008) y no por convicción basada en la congruencia de los ofrecimientos realistas y los ideales políticos que definen las propuestas de los candidatos (Lazo, 2015).

Este panorama no hace sino reflejar el desgaste moral de la sociedad y evidencia la debilidad de la cultura democrática en el país. De este modo, los pobladores del Perú han perdido la esperanza en la clase política, que ya no representan los intereses sociales. Aunque este panorama es similar en otros países de la región (Torres y Zubieta, 2015), en el Perú -y puntualmente en Arequipa- es importante valorar el impacto de la esperanza en la política, mediada por las actitudes de los habitantes. Con esa finalidad, la 
presente investigación ha evaluado, en una muestra de la ciudad de Arequipa, la esperanza y las actitudes hacia la política a través de sus dimensiones de gobernabilidad y participación política, generando un modelo teórico en el que intervienen, además, variables sociodemográficas como el sexo, el grado de instrucción y el estado civil.

En primer término, la esperanza y la participación política han tenido valores altos entre la muestra evaluada. Sin embargo, la gobernabilidad ha tenido valores medios, lo que sugiere que las personas tienen una valoración poco favorable de la gobernabilidad en el país que recae sobre aspectos políticos y de gestión de las instituciones del Estado (Bobbio, 2010). En ese sentido, diversos estudios han puesto de manifiesto una serie de falencias en las instituciones públicas cuando se las compara con sus análogas del sector privado, tanto en la capital (Loli et al., 2017) como en la región Arequipa (Arias y Jiménez, 2013; Arias y Masías, 2014).

Estas deficiencias en la gestión pública han sido explicadas de diversas formas, que comprenden la escasa capacidad técnica de quienes ocupan los cargos públicos, la corrupción en las instituciones nacionales, las limitadas condiciones laborales de los trabajadores del sector y la incomprendida noción de la burocracia en las organizaciones estatales, que terminan afectando la eficiencia y la eficacia de las instituciones del Estado (Ganoza y Stiglich, 2015).

En cuanto al sexo, no se registraron diferencias en función del sexo en ninguna de las variables de estudio, a pesar de que estudios realizados en Lima han reportado que las mujeres tienen mayor nivel de esperanza que los varones (Caycho et al., 2016) y la literatura propia de la psicología política señala que son los varones quienes presentan mayor participación política y tienen actitudes más favorables hacia la política y los asuntos de gobierno (González y Darias, 1998; Espinosa, 2008; Guevara y Espinosa, 2014).

Por otro lado, los casados, convivientes y viudos obtuvieron puntuaciones más altas en esperanza que quienes son solteros y divorciados; vale decir que quienes han tenido o tienen la experiencia favorable de una vida en pareja son los que tienen mayor nivel de esperanza, dato cuyas implicancias en el estudio de las relaciones interpersonales son importantes y que se relaciona con estudios previos, donde se señala que los casados presentan mayor nivel de satisfacción con la vida que los solteros en la ciudad de Lima (Alarcón, 2001). En Arequipa, algunos estudios han relevado diversas cualidades de las personas casadas por sobre otras personas con diferentes estados civiles, tanto en los ámbitos económico y social (Riesco y Arela, 2015; Castro et al., 2013) como en el psicológico (Murillo, 2015; Seperak, 2016).

Con respecto al grado de instrucción, tanto en la esperanza como en las actitudes hacia la política y sus dos dimensiones, la tendencia fue que las personas con mayor grado de instrucción tuvieron mayores puntuaciones en estas variables. Este hallazgo es consistente con el hecho de que personas con mayor nivel socioeconómico tienen una 
actitud más favorable hacia la política (Sabucedo y Fernández, 2001), pues el grado de instrucción, el estatus social y los ingresos económicos han presentado una covariabilidad altamente significativa (Castro et al., 2013). Una explicación a este hecho podría ser que el grado de instrucción implica una mayor formación política o, al menos, un mayor nivel de información o comprensión de los fenómenos políticos (Arellano, 2015; Lazo, 2015; Sorribas y Brussino, 2013), y por otro lado, un mejor posicionamiento económico y social que aportaría a un estado de satisfacción presente que mediaría en un mayor nivel de esperanza.

En ese sentido, Martínez (2006) encontró que en 482 sujetos de quince a ochenta años de la ciudad de Lima había niveles de insatisfacción con el país y el deseo por que haya más progreso, justicia y honestidad, destacando que cuanto mejor es la actitud hacia el presente y futuro, mayor es la satisfacción con el país. El nivel socioeconómico es una variable que presenta diferencias significativas en las actitudes hacia el presente y futuro, en favor de las personas de mayor nivel socioeconómico. En este estudio también se reportó que había una correlación negativa entre la actitud hacia el presente y los deseos vinculados con los gobernantes, lo que supone que tener altas expectativas en los gobernantes resulta frustrante. En nuestro estudio, la esperanza no se relacionó con las actitudes hacia la política, ni con la gobernabilidad o la participación política. Esto podría entenderse, como en el caso anterior, como una falta de esperanza en la clase política, que ubica la esperanza en factores internos más que en externos. En tal sentido, la esperanza podría reposar en la confianza en sí mismo o en la propia satisfacción personal, más que en otras variables sociales. Un estudio de Alarcón (2005) encontró, por ejemplo, que la satisfacción con uno mismo tiene efectos positivos en el nivel socioeconómico, lo que sugiere que mientras más satisfactoria es la valoración que hace la persona sobre su vida, tiene una mejor calidad de vida, expresada en el nivel socioeconómico en el que se desenvuelve.

Por otro lado, el análisis path realizado indica que la esperanza y el grado de instrucción tienen efecto positivo en la participación política, lo que explica el $12 \%$ de la gobernabilidad. Esto quiere decir que la esperanza, sumada al grado de instrucción - con el que tiene interacciones significativas-, impactan de manera favorable en la gobernabilidad, lo que supone la conciencia de que el gobierno depende de la participación política de los ciudadanos. Aquellos que tienen mayor nivel de esperanza y mayor grado de instrucción podrían estar más interesados en participar en política de manera más activa, y no solo a través del voto, como suele entenderse la participación política (Rodríguez, 2004).

La esperanza sería, así, un importante motivador de la participación política, que podría explicarse a través de sus vinculaciones con la empatía (Sierra, 2007; Rosler, Cohen-Chen y Halperin, 2015), la resiliencia (Lemay y Ghazal, 2001), el bienestar social 
(Pereyra, 2010), la felicidad (Lyubomirsky, 2010) y la satisfacción con la vida (Yang, Zhang y Kou, 2016), ya que todas estas variables tienen injerencia en las relaciones interpersonales, la conducta prosocial, el locus de control interno y los valores morales (Arias, 2015). De este modo, la esperanza puede considerarse un importante mediador de la participación política en la medida en que haya cierto nivel de preparación y de conciencia social, marcando distancia de la clase política del país en favor de las expectativas personales y la confianza en sí mismo. Esto implica - parafraseando a John F. Kennedy-que las personas que tienen mayor esperanza no se preocupan en lo que el país puede hacer por ellos, sino más bien en lo que ellos pueden hacer por el país.

Es decir que, aunque las condiciones desfavorables en una realidad sociopolítica concreta se asocian con un rechazo a la democracia y el interés por la política (Laca et al., 2011), la esperanza y un mayor grado de instrucción pueden alentar una participación política más activa (Peterson, 1991). Sin embargo, la esperanza por sí sola no se relaciona con las actitudes hacia la política, sino que es necesaria cierta formación y nivel cultural para que la persona desarrolle metas y capacidades que confluyan en acciones orientadas a futuro, con una visión favorable, pero realista, amparada en la autoconfianza y la necesidad socioafectiva de cambio político y progreso social.

Ahora bien, nuestro estudio no está exento de limitaciones teóricas y metodológicas, pues hubiera sido más conveniente considerar más variables psicológicas, como el locus de control, el bienestar social y la satisfacción con la vida, así como otras de orden socioeconómico, como la estabilidad laboral, los ingresos económicos y la estructura familiar. Así, el análisis path realizado nos ofrecería mayor relevancia teórica y cobraría mayor rigor metodológico. Sin embargo, dado que en el país no son comunes los estudios sobre política y emociones positivas, este trabajo podría servir de precedente para futuras investigaciones, dentro del marco de la psicología política positiva.

\section{REFERENCIAS}

Alarcón, R. (2001). Relaciones entre felicidad, género, edad y estado conyugal. Revista de Psicología (Pontificia Universidad Católica del Perú), 19(1), 27-46.

Alarcón, R. (2005). Efectos de los niveles socioeconómicos sobre la felicidad. Teoría e Investigación Psicológica, 14, 91-112.

Arbuckle, J. L. (2003). Amos user's guide. Chicago: Small Waters.

Arellano, R. (2015). Vamos a comprar un político. Aplicando nuestra experiencia de compra a la más importante elección. Lima: Planeta.

Arias, W. L. (2015). Conducta prosocial y psicología positiva. Avances en Psicología, 23(1), 37-47. 
Arias, W. L. (2016). ¿Es la psicología positiva presentista?: breve revisión de las críticas a la psicología positiva a la luz de la historia de la psicología y la epistemología. Revista Peruana de Historia de la Psicología, 2, 93-110.

Arias, W. L. y Jiménez, N. A. (2013). Síndrome de burnout en docentes de educación básica regular de Arequipa. Educación, 22(42), 53-76.

Arias, W. L. y Masías, M. A. (2014). Ocio cibernético en trabajadores de instituciones públicas y privadas de Arequipa. Ciencia \& Trabajo, 16(50), 88-92.

Arias, W. L., Timaná, C., Román, A., Maquera, C., Zúñiga, C. y Díaz Cano, M. (2017). Un cuestionario de actitudes hacia la política validado en habitantes de la ciudad de Arequipa, Perú. Psicologia Política, 17(39), 386-398.

Arias, W. L., Ventura-León, J. L., Caycho-Rodríguez, T., Román, A., Ceballos, K. D., Zúñiga, C., Díaz Cano, M. y Lovón, E. (2018). Estructura interna y consistencia interna del Índice de Esperanza de Herth en habitantes de la ciudad de Arequipa. Revista Argentina de Ciencias del Comportamiento, 10(3), 1-11.

Arnau, R. C., Martínez, P., Niño de Guzmán, I., Herth, K. y Konishi, C. Y. (2010). A SpanishLanguage Version of the Herth Hope Scale: Development and Psychometric Evaluation in a Peruvian Sample. Educational and Psychological Measurement, 70(5), 808-824. doi: 10.1177/0013164409355701

Bobbio, N. (2010). Estado, gobierno y sociedad. Por una teoría general de política. México, D. F.: Fondo de Cultura Económica.

Bronk, K. C., Hill, P. L., Lapsley, D. K., Talib, T. L. y Finch, H. (2009). Purpose, hope, and life satisfaction in three age groups. The Journal of Positive Psychology, 4(6), 500-510. doi: $10.1080 / 17439760903271439$

Buss, A. R. (1979). The emerging field of the sociology of psychological knowledge. In A. Buss, Psychology in Social Context (pp. 1-24). New York: Iwington Publishers.

Cassaretto, M. y Martínez, P. (2009). Validación de la escala del sentido del humor en estudiantes universitarios. Revista de Psicología (Pontificia Universidad Católica del Perú), 27(2), 287-310.

Cassaretto, M. y Martínez, P. (2012). Razones para vivir en jóvenes adultos: validación del RFL-YA. Revista de Psicología (Pontificia Universidad Católica del Perú), 30(1), 169-188.

Castilla, H., Urrutia, C. M., Shimabukuro, M. y Caycho, T. (2014). Análisis psicométrico del Índice de Esperanza de Herth en una muestra no clínica peruana. Psicología desde El Caribe, 31(2), 187-206.

Castro, R., Arias, W. L., Domínguez, S., Masías, M. A., Salas, X., Canales, F. y Flores, A. (2013). Integración familiar y variables socioeconómicas en Arequipa metropolitana. Revista de Investigación, 4, 35-65. 
Caycho, T., Castilla, H. y Ventura-León, J. L. (2016). Esperanza en adolescentes y jóvenes peruanos: diferencias según el sexo y la edad. Psychologia. Avances de la Disciplina, 10(2), 33-41.

Chacón, E. y Alvarado, J. M. (2007). Evaluación de las actitudes que definen la participación política. Psicología, 26(1), 6-21.

Cheavens, J. S., Feldman, D. B., Woodward, J. T. y Snyder, C. R. (2006). Hope in cognitive psychotherapies: On working with client strengths. Journal of Cognitive Psychotherapy: An International Quarterly, 20(2), 135-145. doi: 10.1891/ jcop.20.2.135

Cleary, M., Sayers, J. M. y López, V. (2016). Hope and Mental Health Nursing. Issues in Mental Health Nursing, 37(9), 692-694. doi: 10.1080/01612840.2016.1221676.

Dargent, E. (2015). Crimen y prensa en Lima: Análisis del papel de la prensa escrita durante una "ola" de secuestros (septiembre-octubre 2003). Revista de Ciencia Política y Gobierno, 2(3), 9-32.

Dávila, J. M., Fouce, J. G., Gutiérrez, L., Lillo., A. y Martín, E. (1998). La psicología política contemporánea. Psicología Política, 17, 21-43.

Delfino, G. y Zubieta, E. (2014). Participación política pacífica y agresiva. Psicología Política, 48, 25-46.

Dufault, K. y Martocchio, B. (1985). Hope: Its spheres and dimensions. Nursing Clinics of North America, 20, 379-391.

Eiroa, F. J. (2013). Cambio sociocultural y bienestar psicosocial. Psicología Política, 47, 39-53.

Espinosa, A. (2008). Decidiéndose por el mal menor. Psicología Política, 37, 47-70.

Espinosa, A., Acosta, Y., Valencia, J., Vera, A., Soares, A., Romero, J. C. y Beramendi, M. (2016). Calidez, competencia, moralidad y nacionalismo ideal como dimensiones autoestereotípicas del autoconcepto nacional en seis países de Latinoamérica. Avances en Psicología Latinoamericana, 34(2), 395-413.

Espinosa, A., Beramendi, M. y Zubieta, E. (2015). Identidad nacional y bienestar social: una síntesis meta analítica de estudios en Argentina, México y Perú. Revista Interamericana de Psicología, 49(1), 27-39.

Espinosa, A., Cueto, R. M. y Schmitz, M. (2012). De una psicología social de fenómenos políticos al desarrollo de la psicología política en el Perú. Psicologia Política, 12(25), 465-479.

Espinosa, A., Freire, S. y Ferrándiz, J. (2016). Identificación colectiva y bienestar en una comunidad rural de la costa norte del Perú. Revista de Psicología (Pontificia Universidad Católica del Perú), 34(1), 201-209. 
Espinosa, A. y Tapia, G. (2011). Identidad nacional como fuente de bienestar subjetivo y social. Boletín de Psicología 102, 71-87.

Franco, C. (1980). Imagen de la sociedad, valoración de la participación política y personalidad. Revista Latinoamericana de Psicología, 12(2), 277-292.

Frankl. V. (2002). El hombre en busca de sentido. 21. ${ }^{a}$ edición. Barcelona: Herder.

Fromm, E. (2012). La revolución de la esperanza. México, D. F.: Fondo de Cultura Económica.

Ganoza, C. y Stiglich, A. (2005). El Perú está calato. El falso milagro de la economía peruana y las trampas que amenazan nuestro progreso. Lima: Planeta.

Gattino, S., Roccato, M. y Tamagnone, L. (2002). Percepción de la realidad social y afiliación política. Psicología Política, 24, 7-29.

Goertzel, T. (2005). Corruption, leadership and development in Latin America. Psicología Política, 31, 77-102.

González, M. y Darias, M. (1998). Predicción de la conducta de voto. Psicología Política, 17, 45-78.

Gordillo, F.; Arana, J. M.; Mestas, L. y Salvador, J. (2012). Compatibilidad y confianza entre votante y candidato. Psicología Política, 45, 27-41.

Guevara, L. A. y Espinosa, A. (2014). Estereotipos y emociones intergrupales en Facebook durante las elecciones generales peruanas del 2011. Revista Electrónica de Psicología Política, 13(33), 25-48.

Hernández, R., Fernández, C. y Baptista, P. (2006). Metodología de la investigación. México, D. F.: McGraw-Hill.

Herth, K. (1991). Development and refinement of an instrument to measure hope. Scholarly Inquiry for Nursing Practice, 5(1), 39-51.

Herth, K. (1992). Abbreviated instrument to measure hope: development and psychometric evaluation. Journal of Advanced Nursing, 17(10), 1251-1259. doi: 10.1111/j.13652648.1992.tb01843.x

Ibáñez, T. (1993). La dimensión política de la psicología social. Revista Latinoamericana de Psicología, 25(1), 19-34.

Jiménez, F. (1993). Freud y la política. Revista Latinoamericana de Psicología, 25(1), 105-113.

Laca, F. A., Santana, H., Ochoa, Y. y Mejía, J. C. (2011). Percepción del bienestar social, anomia, interés e impotencia política en relación con las actitudes hacia la democracia. Liberabit, 17(1), 7-18.

Lazo, S. (2015). Comportamiento electoral en el Perú: un análisis del rol de las variables sociodemográficas y socioeconómicas en las elecciones presidenciales en primera vuelta de 2006 y 2011. Revista de Ciencia Política y Gobierno, 2(3), 51-80. 
Lemay, R. y Ghazal, H. (2001). Resilience and Positive Psychology: Finding Hope. Child \& Family, 5(1), 10-21.

León, F. y León, F. R. (2014). Un experimento para evaluar las reacciones emocionales según la orientación a la dominación social y el éxito diplomático en el Perú. Revista de Psicología de Arequipa, 4(1), 47-54.

Loli, A., Navarro, V., Del Carpio, J., Vergara, A., Castillo, D., Espinoza, A. y Borja, 0. (2017). El contrato psicológico, el desempeño y la satisfacción en empleados de organizaciones públicas y privadas de Lima. Revista de Investigación en Psicología, 20(1), 61-78. doi: 10/15381/rinvp.v20i1.13352

López, S. J., Sage, R., Robinson, C., Marques, S. C. y Ribeiro, J. P. (2014). Measuring and promoting hope in shoolchildren. In M. Furlong, R. Gilman y E. S. Huebner (eds.), Handbook of Positive Psychology in Schools (pp. 37-50). London: Routledge.

Lyubomirsky, S. (2010). La ciencia de la felicidad. Buenos Aires: Urano.

Malik, A. (2013). Efficacy, hope, optimism and resilience at workplace - Positive Organizational Behavior. International Journal of Scientific and Research Publications, 3(10), 1-4.

Martínez, P. (2006). Perspectiva futura del Perú y el nivel de satisfacción con él. Liberabit, $12,113-121$.

Martínez, P., Cassaretto, M. y Herth, K. (2012). Propiedades psicométricas de la Escala de Esperanza de Herth en español. Revista Iberoamericana de Diagnóstico y Evaluación Psicológica, 33(1), 127-145.

Mentinis, M. (2011). ¿De qué te ríes? Hacia una crítica psicosocial del humor y de la risa en la política radical. Teoría y Crítica de la Psicología, 1, 131-141.

Montero, M. (1991). Una orientación para la psicología política en América Latina. Psicología Política, 3, 27-43.

Montero, M. y Dorna, A. (1993). La psicología política: una disciplina en la encrucijada. Revista Latinoamericana de Psicología, 25(1), 7-14.

Morales, J. F., Gaviria, E., Molero, F., Arias, A. y Páez, D. (2000). Individualism: One or many? Psicothema, 12(Supl.), 34-44.

Murillo, M. G. (2015). Desarrollo del juicio moral en personas casadas y divorciadas en la ciudad de Arequipa. Avances en Psicología, 23(1), 73-85.

Park, N., Peterson, C. y Sun, J. K. (2013). La psicología positiva: investigación y aplicaciones. Terapia Psicológica, 31(1), 11-19.

Pasquino, G. (2001). Ciudadanía mundial. Psicología Política, 23, 59-75.

Peterson, C. y Seligman, M. E. P. (2004). Character, strengths and virtues: A handbook and classification. Washington, D. C.: American Psychological Association. 
Peterson, S. A. (1991). "No te preocupes, sé feliz": satisfacción con la vida y política. Psicología Política, 3, 65-75.

Pereyra, M. (2010). Esperanza, salud y bienestar. Avances en Psicología, 18(1), 9-31.

Polo, L., Godoy, J. C., Imhoff, D. y Brussino, S. (2014). Following tracks of an emerging área: bibliometric analysis of Latin American Political Psychology in the 20002010 period. Universitas Psychologica, 13(4), 15-25.

Quintana, D. y Curbelo, L. (2015). La psicología y las políticas: entre lo individual y lo público. Alternativas Cubanas en Psicología, 9, 15-23.

Riesco, R. y Arela, R. (2015). Impacto de la estructura familiar en la satisfacción con los ingresos en los hogares urbanos en Perú. Economía, 38(76), 51-76.

Riesco, G., Alpaca, L. y Arias, W. L. (2015). Evolución del pago de coimas a la policía en el Perú entre los periodos 2005-2006 y 2012-2013 y su impacto en la economía familiar. Revista de Investigación, 6, 77-99.

Rodríguez, A. (2001). La psicología social y la psicología política latinoamericana: ayer y hoy. Psicología Política, 22, 41-52.

Rodríguez, D., Mirón, L., Godás, A. y Serrano, G. (1996). Valores y participación política en los adolescentes españoles. Psicología Política, 12, 7-33.

Rodríguez, G. (2004). Significado de la participación política en habitantes del Valle de Méjico. Psicología Política, 29, 69-78.

Romagosa, J. E. y Albizú-Miranda, C. (1986). La fe, la esperanza y el amor y su relación con la salud mental. Revista Latinoamericana de Psicología, 18(3), 451-456.

Rosler, N., Cohen-Chen, S. y Halperin, E. (2015). The Distinctive Effects of Empathy and Hope in Intractable Conflicts. Journal of Conflict Resolution, 61(1), 114-139. doi: $10.1177 / 0022002715569772$

Rottenbacher, J. M. (2015). Trato discriminatorio hacia empleadas domésticas y segregación socioespacial en balnearios de Lima. Revista de Psicología (Pontificia Universidad Católica del Perú), 33(2), 241-275.

Rottenbacher, J. M. y De la Cruz, M. (2012). Ideología política y actitudes hacia la minería en el Perú: Entre el crecimiento económico, el respeto por las formas de vida tradicionales y el ambientalismo. Liberabit, 18(1), 83-96.

Sabucedo, J. M. y Fernández, C. (2001). Elementos psicosociales en la conducta de voto nacionalista. Psicothema, 13(2), 181-185.

Salgado, C. (1996). Construcción de una escala para medir la actitud hacia la identidad nacional. Veritas, 2, 29-37.

Salgado, C. (1997). La identidad nacional: una aproximación a través de las actitudes en estudiantes del último año de psicología de dos universidades particulares de Lima. Veritas, 3, 21-30. 
Sartore, A. C., Alvez, S. A. y Herth, K. (2010). Cultural adaptation and validation of the Herth Hope Index for Portuguesse language: study in patients with chronic illnes. Texto \& Contexto Enfermagem, 19(4), 754-761. doi: 10.1590/S0104-07072010000400019

Seperak, R. (2016). Motivos extrínsecos-intrínsecos y trascendentes en la decisión de contraer matrimonio en casados, separados y/o divorciados. Revista de Psicología de Arequipa, 6(1), 349-368.

Sierra, G. (2007). ¿Se relacionan la esperanza y la empatía? Psicología Iberoamericana, 15(2), 2-10.

Silva, L. C. (2013). Ética das virtudes e liberalismo político: sobre a tolerancia. Synesis, 5(2), 113-135.

Snyder, C. R. (2000). Past and possible futures of hope. Journal of Social and Clinical Psychology, 19(1), 11-28. doi: 10.1521/jscp.2000.19.1.11

Snyder, C. R., Rand, K. L. y Sigmon, D. R. (2002). Hope theory: a member of the positive psychology family. En C. R. Snyder y S. Lopez (eds.), Handbook of Positive Psychology (pp. 257-276). New York: Oxford University Press.

Sorribas, P. M. y Brussino, S. (2013). Participación política orientada al sistema representativo. Psicología Política, 47, 91-112.

Schwartz, S. H. y Barnea, M. (1995). Los valores en las orientaciones políticas. Psicología Política, 11, 15-40.

Temkin, B. y Flores, G. (2011). Importancia del autoritarismo-dogmatismo en las actitudes sociopolíticas. Psicología Política, 43, 65-84.

Torres, C. M. y Zubieta, E. M. (2015). Consenso y divergencias en las representaciones sociales de la dirigencia política. Revista de Psicología (Pontificia Universidad Católica del Perú), 33(1), 87-129.

Valdez, J. L., Maya, M. U., Aguilar, Y. P., Bustillos, R., Antonio, P., Valdés, A. y González, N. I. (2014). Desesperanza en jóvenes: un análisis por sexo. Avances en Psicología, 22(2), 221-232.

Yager-Elorriaga, D., Berenson, K. y McWhirter, P. (2014). Hope, ethnic pride, and academic achievement: Positive Psychology and Latino Youth. Psychology, 5, 1206-1214.

Yang, Y., Zhang, M. y Kou, Y. (2016). Self-compassion and life satisfaction: The mediating role of hope. Personality and Individual Differences, 98, 91-95. doi: 10.1016/j. paid.2016.03.086 
